\title{
Defectos del suelo pélvico posparto en función del índice de masa corporal pregestacional y ganancia ponderal de la mujer durante el embarazo
}

\author{
Marta Moreno López, ${ }^{1}$ (D) Marina de la Plata Daza, ${ }^{2}$ (D) Sara Rojo Novo, ${ }^{1}$ \\ Zoraida Frías Sánchez, ${ }^{3}$ (D) Manuel Pantoja Garrido. ${ }^{1}$
}

\section{RESUMEN}

\begin{abstract}
Objetivo: Determinar si existe asociación causal entre el índice de masa corporal materno pregestacional o la ganancia ponderal, y la presencia de disfunciones de suelo pélvico posparto, como la incontinencia urinaria, fecal y la disfunción sexual.

Métodos: Estudio observacional prospectivo de cohorte, con 500 gestantes controladas en el Hospital Universitario Virgen Macarena (Sevilla), durante el primer trimestre de 2012. Se categorizaron según su indice de masa corporal pregestacional y su ganancia ponderal durante el embarazo. Se analizaron las causas de la presencia de incontinencia urinaria, fecal y disfunción sexual a los 6, 12 meses y a los 7 años del parto.

Resultados: Se observó sobrepeso en el $26 \%$, obesidad en el 16,6\% y ganancia ponderal excesiva en el 46,7\%. La macrosomía fetal se asoció con incontinencia urinaria y disfunción sexual en los tres periodos estudiados y con incontinencia fecal a los 7 años. Se obtuvieron asociaciones estadísticamente significativas entre obesidad e incontinencia urinaria a los 7 años (OR 2,737 IC 95 \%: 1,262-5,938) y disfunción sexual a los 12 meses y a los 7 años (OR 7,321 IC95\%: 1,307-41,006) y con la ganancia ponderal, (OR: 8,427 IC 95\%: 1,026-69,225)

Conclusiones: La gestación y el parto son elementos de riesgo de las disfunciones de suelo pélvico, encontrándose asociados a su desarrollo factores como la macrosomía fetal, la obesidad pregestacional y la ganancia ponderal excesiva. Es por ello importante el asesoramiento a las pacientes sobre el control de peso previo y durante la gestación.
\end{abstract}

Palabras clave: Obesidad, Ganancia ponderal, Índice de masa corporal, Gestación, Defectos suelo pélvico.

\section{Postpartum pelvic floor defects according to pre-gestational body mass index and women's weight gain during pregnancy SUMMARY}

Objective: To determine if there is a causal association between the pre-pregnancy maternal body mass index or weight gain, and the presence of postpartum pelvic floor dysfunctions, such as urinary and fecal incontinence and sexual dysfunction. Methods: We carried out a prospective observational cohort study with 500 pregnant women controlled at HUV Macarena (Seville) from january to april of 2012. Patients were categorized according to their pregestational BMI and the weight gain during pregnancy. We analyzed the causes of the presence of urinary and fecal incontinence and sexual dysfunction at 6,12 months and 7 years after delivery.

Results: Overweight was observed in $26 \%$, obesity in 16,6\% and excessive weight gain in 46,7\%. Fetal macrosomia was associated with urinary incontinence and sexual dysfunction in all three periods and with fecal incontinence at 7 years. Statistically significant associations were observed between urinary incontinence at 7 year and obesity, OR 2,737 IC 96\% (1,262-5,938) and between sexual dysfunction at 1 year and at 7 years with obesity, OR 7,321 IC $95 \%(1,307-41,006)$ and with weight gain, OR 8,427 IC 95\% (1,026-69,225).

Conclusions: Pregnancy and childbirth are risk factors for pelvic floor dysfunctions, since fetal macrosomia, pre-pregnancy obesity and excessive weight gain have been associated with their development. It is important to advise patients on weight control before and during pregnancy.

Keywords: Obesity, Gestational weight gain, Body mass index, Pregnancy, Pelvic floor disorders.

\begin{abstract}
'Unidad de Gestión Clínica de Obstetricia y Ginecología del Hospital Universitario Virgen Macarena de Sevilla, (España). ${ }^{2}$ Unidad de Gestión Clínica de Obstetricia y Ginecología del Hospital Universitario de La Merced de Osuna (Sevilla, España). ${ }^{3}$ Unidad de Gestión Clínica de Ginecología y Patología Mamaria del Hospital Universitario Virgen del Rocío de Sevilla, (España). Correo para correspondencia: marta.m191@gmail.com
\end{abstract}

Forma de citar este artículo: Moreno López M, De la Plata Daza M, Rojo Novo S, Frías Sánchez Z, Pantoja Garrido M. Defectos del suelo pélvico posparto en función del índice de masa corporal pregestacional y ganancia ponderal de la mujer durante el embarazo. Rev Obstet Ginecol Venez. 2022; 82(1): 73-82. https://doi.org/10.51288/00820110

\section{INTRODUCCIÓN}

Las disfunciones del suelo pélvico engloban diversas alteraciones clínicas relacionadas con cambios anatómicos y funcionales que derivan en trastornos tales como la incontinencia urinaria, la incontinencia fecal, el prolapso de órganos pélvicos o las disfunciones 
sexuales (1). Estas patologías tienen una prevalencia alta (especialmente en mujeres) y conllevan unas implicaciones socioeconómicas importantes, con un impacto considerable en la calidad de vida de las pacientes (2). La evolución de estas disfunciones es un proceso complejo secundario a múltiples factores. Estados como la gestación y el puerperio se correlacionan directamente con el agravamiento de estos problemas $(1,3)$ ya que se ha observado que en su fisiopatología intervienen factores hormonales y mecánicos acontecidos durante la gestación, parto y puerperio, aunque estos cambios tienden a restablecerse gradualmente, con una regresión al estado pregestacional en un periodo medio de unos 12 meses. Existen también numerosos estudios que han demostrado que tanto la gestación como el parto están asociados, en ocasiones, a traumatismos y sobrecargas de las estructuras del suelo pélvico que generan secuelas permanentes en las pacientes, siendo el parto vaginal el factor de riesgo más relacionado con estas disfunciones $(2,4)$. Por otro lado, los desgarros perineales y la episiotomía pueden producir daño a nivel de los músculos, nervios y tejido conjuntivo del suelo pélvico, así como desencadenar una disminución en la fuerza muscular y capacidad de continencia durante el posparto (5). Además, se ha objetivado que el efecto perjudicial sobre el suelo pélvico es más pronunciado en los partos instrumentados, sobre todo mediante fórceps, además de otros factores de riesgo como el peso fetal, una segunda fase de parto prolongada o un índice de masa corporal (IMC)/ganancia ponderal excesivos de la paciente (4).

Diversos son los estudios que han relacionado el sobrepeso y la obesidad con la incontinencia urinaria de esfuerzo y con el prolapso de órganos pélvicos, demostrando que un IMC elevado predice una mayor gravedad de los síntomas. Algunos autores igualan su importancia en la fisiopatología de las disfunciones del suelo pélvico con la de la historia obstétrica. Además, se ha observado que la obesidad se asocia a un mayor tiempo quirúrgico en las técnicas anti-incontinencia y a una mayor tasa de incontinencia de urgencia posterior en comparación con pacientes con IMC normal. Este es un problema fácilmente modificable con cambios en el estilo de vida, ya que se ha demostrado la mejoría clínica de la incontinencia urinaria con la pérdida de peso $(6,7)$.

Para poder conocer y cuantificar la intensidad y severidad de los síntomas derivados de las disfunciones de suelo pélvico, los cuestionarios validados suponen un instrumento de bajo costo, no invasivo y de fácil reproductibilidad, que ayudan a conocer el impacto de estas alteraciones en la calidad de vida de las pacientes (1). Por tanto, el objetivo de este trabajo fue determinar si en la población gestante atendida en el Hospital Universitario Virgen Macarena de Sevilla, existe una asociación entre el IMC materno pregestacional elevado o la ganancia ponderal excesiva, y la presencia de disfunciones de suelo pélvico; así como, conocer si el tipo y vía del parto, el peso del recién nacido o la existencia de desgarros perineales y/o episiotomía se asocian a mayores tasas de incontinencia urinaria, fecal y/o disfunción sexual. Todo ello, con el fin de estudiar medidas que favorezcan la reducción en las tasas de dichos defectos.

\section{MÉTODOS}

Estudio observacional prospectivo, de cohorte a partir de 500 gestantes que fueron controladas y atendidas en el Hospital Universitario Virgen Macarena de Sevilla (España). Se incluyeron a todas las gestantes que ingresaron para finalizar la gestación, bien por trabajo de parto establecido, inducción del parto o cesárea electiva, desde enero hasta abril de 2012. La información se recopiló a través de la historia clínica y la entrevista personal a la paciente durante el ingreso y posparto inmediato, acerca de su IMC pregestacional (calculado mediante la talla y el peso obtenidos en la primera consulta de control de la gestación), la existencia de patología previa a la gestación, 


\section{DEFECTOS DEL SUELO PÉLVICO POSPARTO EN FUNCIÓN DEL ÍNDICE DE MASA CORPORAL PREGESTACIONAL Y GANANCIA PONDERAL DE LA MUJER DURANTE EL EMBARAZO}

antecedentes obstétricos (número de partos, cesáreas, abortos, gestaciones ectópicas, etc.), desarrollo de patología durante la gestación y mecanismo de inicio y fin del parto. Además, se recogieron los datos del tipo de parto, duración de este, sexo y peso de recién nacido, test de apgar o presencia de desgarro perineal, episiotomía, etc. Se excluyeron 10 mujeres por falta de alguno de los datos en su historia, obteniendo un tamaño muestral inicial de 490 pacientes, que se redujo a 366 tras la fase de entrevista. Dicha entrevista se realizó vía telefónica a todas las pacientes, pasando cuestionarios validados sobre incontinencia urinaria (ICQ: International Consultation on Incontinence Questionnaire), incontinencia fecal (test de Wexner) y satisfacción sexual (PISQ-12 modificado) a los 6 meses, a los 12 meses y a los 7 años del parto.

- La escala ICQ identifica a las pacientes con incontinencia de orina, además, del impacto en su calidad de vida. Consta de 4 ítems y su puntuación se obtiene mediante la suma de los tres primeros, considerando como diagnóstico de incontinencia urinaria toda puntuación mayor a cero.

- La escala de Wexner evalúa la incontinencia fecal en sus distintas modalidades (gases, líquida y sólida), así como la afectación que produce en la calidad de vida de la paciente. Tiene un sistema de puntuación que evalúa la gravedad de la incontinencia fecal, asociando una puntación de 0 a la continencia perfecta y de 20 , a una incontinencia fecal completa.

- La escala PISQ-12 evalúa la función sexual en mujeres con prolapso genital y/o incontinencia urinaria, siendo el primer sistema validado $\mathrm{y}$ adaptado culturalmente para su uso en España. En este estudio se utilizó esta escala modificada, realizando únicamente las preguntas sobre función sexual relacionadas con las disfunciones de suelo pélvico (incontinencia o prolapso).
Para el análisis de los datos se planteó un estudio estadístico descriptivo con la media, desviaciones estándar y porcentaje de las variables generales de las pacientes, analizando la edad, gestaciones previas, IMC, ganancia ponderal, tiempo de amenorrea y de expulsivo, tipo de parto, peso del recién nacido, presencia de episiotomía y de desgarro perineal. Además, se estableció si la ganancia ponderal fue adecuada o excesiva, ajustándola al IMC pregestacional, según las tablas recomendadas por la Sociedad Española de Ginecología y Obstetricia (SEGO) y se categorizó como macrosomía a los recién nacidos cuyo peso fue mayor a 4000 g. Además, se analizaron las tasas observadas de los tres tipos de defectos (incontinencia urinaria, fecal y disfunción sexual) en los tres periodos estudiados (6 meses, 12 meses y 7 años posparto).

Una vez realizado el análisis descriptivo se realizó una regresión logística binaria, con el fin de valorar la relación entre las disfunciones de suelo pélvico y la ganancia de peso excesiva durante la gestación, así como con el IMC pregestacional. Además, se incluyeron en el análisis otras variables independientes (macrosomía fetal, tipo de parto, presencia de desgarro perineal, realización de episiotomía y tiempo de expulsivo) que podían actuar como variables de confusión para los defectos de suelo pélvico estudiados. Inicialmente, se realizó una regresión logística individual con cada una de las variables $y$, posteriormente, un análisis conjunto de todas las variables estudiadas. El análisis se repitió con los tres tipos de disfunción de suelo pélvico (incontinencia de orina, fecal y disfunción sexual) en los tres periodos estudiados (6 meses, 12 meses y 7 años). Se consideró la significación estadística de los resultados con valores de $\mathrm{p}<0,05$. Se utilizó el programa SPSS 21 para Mac para el análisis estadístico.

Los procedimientos seguidos cumplieron las normas éticas del Comité de Experimentación Humana responsable, de acuerdo con la Asociación Médica Mundial y la Declaración de Helsinki, siguiéndose 
los protocolos del centro sobre la publicación de datos de pacientes, asegurando la confidencialidad de los mismos.

\section{RESULTADOS}

El tamaño muestral inicial fue de 500 pacientes, que se redujo a 366 tras la fase de entrevistas. La edad media fue de 30,40 años, con una edad mínima de 17 y una máxima de 46 años. El número medio de gestaciones fue de 1,82, siendo la mayoría de las pacientes primigestas. Atendiendo al índice de masa corporal previo a la gestación, un 54,6\% presentaba normopeso (IMC $18,5-25 \mathrm{Kg} / \mathrm{m}^{2}$ ), un $26 \%$ sobrepeso (IMC $25-30 \mathrm{Kg} / \mathrm{m}^{2}$ ) y un $16,6 \%$ partían de un IMC mayor de $30 \mathrm{Kg} / \mathrm{m}^{2}$. La ganancia ponderal media fue de $13,89 \mathrm{Kg}$, oscilando desde la pérdida de hasta 15 $\mathrm{Kg}$ a la ganancia de 32. La ganancia ponderal ajustada al IMC previo a la gestación fue adecuada en el 53,3\% de las pacientes y excesiva en el 46,7 \%.

Analizando los datos de la gestación en curso, el tiempo de amenorrea medio fue de 39 semanas y 4 días, siendo el tiempo medio de expulsivo de 1 hora y 35 minutos. La finalización de la gestación en un $62,3 \%$ de los casos fue mediante parto eutócico, en un $10,9 \%$ mediante ventosa, un 5,2\% mediante fórceps, un 1,6 \% espátulas y en un 19,9\% de los casos mediante cesárea. El peso medio de los recién nacidos fue de 3315,5 g (2015 g - $4825 \mathrm{~g}$ ), con una tasa de macrosomía (peso del recién nacido mayor o igual a $4000 \mathrm{~g}$ ) del 6,3\%. En cuanto a la episiotomía, se realizó en un 32,4\% de las pacientes. Se observó desgarro perineal en un 47,7 \% de las encuestadas. Así mismo, se analizaron las tasas de los tres defectos de suelo pélvico estudiados (incontinencia de orina, fecal y disfunción sexual), que se pueden observar resumidos en la tabla 1.

Posteriormente, se realizó una regresión logística, cuyos datos se resumen en las tablas 2, 3 y 4 .
Tabla 1. Tasas de incontinencia urinaria, fecal y disfunción sexual a los 6 meses, 12 meses y 7 años del parto

\begin{tabular}{lcccc}
\hline Variables & & 6 meses & 12 meses & 7 años \\
\hline $\begin{array}{l}\text { Incontinencia } \\
\text { urinaria (\%) }\end{array}$ & No & 85,7 & 86,2 & 87,7 \\
& No & 99 & 99,2 & 99,2 \\
$\begin{array}{l}\text { Incontinencia } \\
\text { fecal (\%) }\end{array}$ & Sí & 1 & 0,8 & 0,8 \\
$\begin{array}{l}\text { Disfunción } \\
\text { sexual (\%) }\end{array}$ & No & 97,8 & 98,1 & 98,1 \\
\hline
\end{tabular}

Comenzando por el análisis de la incontinencia de orina se observó que se obtuvo significación estadística con la presencia de macrosomía fetal, con una OR de 8,264 IC $95 \%(3,421-19,964)$ a los 6 meses del parto; OR de 7,145 IC $95 \%(2,954-17,286)$ a los 12 meses y OR: 3,512 IC $95 \%(1,358-9,080)$ a los 7 años. También se observaron resultados estadísticamente significativos entre la presencia de obesidad y la incontinencia de orina a los 7 años, con una OR: 2,727 IC 95 \% (1,262$5,938)$, mientras que con el resto de las variables no se obtuvo la significación estadística. Analizando el tipo de parto, se observó que, en los tres periodos estudiados, el parto eutócico, el uso de ventosa y el de fórceps presentaron una asociación positiva con la incontinencia de orina, si se compara con la cesárea. Ocurrió lo mismo con la presencia de desgarro y episiotomía, encontrando una asociación positiva con la presencia de incontinencia de orina en los tres periodos de estudio.

Como ya se comentó anteriormente, en cuanto a las variables asociadas con el peso materno (la presencia de sobrepeso u obesidad previa al parto y la ganancia ponderal excesiva durante la gestación), solo se obtuvo significación estadística entre la obesidad y la presencia de incontinencia de orina a los 7 años. No obstante, en 


\section{DEFECTOS DEL SUELO PÉLVICO POSPARTO EN FUNCIÓN DEL ÍNDICE DE MASA CORPORAL PREGESTACIONAL Y GANANCIA PONDERAL DE LA MUJER DURANTE EL EMBARAZO}

Tabla 2. Regresión logística binomial entre la incontinencia urinaria a los 6 meses, 12 meses y 7 años del parto y las variables independientes

\begin{tabular}{|c|c|c|c|c|c|c|c|}
\hline \multirow{2}{*}{\multicolumn{2}{|c|}{ Variable }} & \multicolumn{2}{|r|}{6 meses posparto } & \multicolumn{2}{|c|}{12 meses posparto } & \multicolumn{2}{|c|}{7 años posparto } \\
\hline & & $\mathrm{p}$ & OR & $\mathrm{p}$ & OR & $\mathrm{p}$ & OR \\
\hline Macrose & mía & 0 & $8,264(3,421-19,964)$ & 0 & $7,145(2,954-17,286)$ & 0,01 & $3,512(1,358-9,080)$ \\
\hline \multirow{4}{*}{$\begin{array}{l}\text { Tipo de } \\
\text { parto }\end{array}$} & Eutócico & 0,584 & $1,246(0,567-2,738)$ & 0,448 & $1,375(0,604-3,128)$ & 0,059 & $2,816(0,961-8,252)$ \\
\hline & Ventosa & 0,689 & $1,255(0,412-3,822)$ & 0,534 & $1,434(0,460-4,469)$ & 0,101 & $3,044(0,805-11,512)$ \\
\hline & Fórceps & 0,691 & $1,333(0,323-5,499)$ & 0,565 & $1,523(0,363-6,399)$ & 0,149 & $3,234(0,658-15,903$ \\
\hline & Espátulas & 0,999 & 0 & 0,999 & 0 & 0,999 & 0 \\
\hline \multicolumn{2}{|c|}{ Desgarro } & 0,61 & $1,166(0,645-2,109)$ & 0,418 & $1,283(0,702-2,345)$ & 0,332 & $1,368(0,727-2,575)$ \\
\hline \multicolumn{2}{|c|}{ Episiotomía } & 0,427 & $1,283(0,693-2,375)$ & 0,308 & $1,382(0,742-2,574)$ & 0,107 & $1,697(0,893-3,224)$ \\
\hline \multicolumn{2}{|c|}{ Sobrepeso } & 0,36 & $1,382(0,692-2,763)$ & 0,243 & $1,519(0,753-3,065)$ & 0,228 & $1,588(0,749-3,369)$ \\
\hline \multirow{3}{*}{\multicolumn{2}{|c|}{$\begin{array}{l}\text { Obesidad } \\
\text { Ganancia ponderal } \\
\text { excesiva } \\
\text { Tiempo expulsivo }\end{array}$}} & 0,122 & $1,82(0,853-3,885)$ & 0,076 & $2,000(0,929-4,306)$ & 0,011 & $2,737(1,261-5,938)$ \\
\hline & & 0,382 & $1,301(0,722-2,345)$ & 0,386 & $1,303(0,716-2,372)$ & 0,245 & $1,457(0,773-2,747)$ \\
\hline & & 0,839 & $1(0,995-1,004)$ & 0,966 & $1,000(0,996-1,004)$ & 0,729 & $0,999(0,995-1,004)$ \\
\hline
\end{tabular}

Entre paréntesis se muestran los intervalos de confianza al $95 \%$ para cada OR (Odds ratio)

Tabla 3. Regresión logística binomial entre la incontinencia fecal a los 6 meses, 12 meses y 7 años del parto y las variables independientes

\begin{tabular}{|c|c|c|c|c|c|c|c|}
\hline \multirow{2}{*}{\multicolumn{2}{|c|}{ Variable }} & \multicolumn{2}{|c|}{6 meses posparto } & \multicolumn{2}{|r|}{12 meses posparto } & \multicolumn{2}{|r|}{7 años posparto } \\
\hline & & $\mathrm{p}$ & OR & $\mathrm{p}$ & OR & $\mathrm{p}$ & OR \\
\hline \multicolumn{2}{|c|}{ Macrosomía } & 0,163 & $5,152(0,515-51,582)$ & 0,100 & $7,750(0,676-88,815)$ & 0,005 & $32,571(2,837-373,903)$ \\
\hline \multirow{4}{*}{$\begin{array}{l}\text { Tipo de } \\
\text { parto }\end{array}$} & Eutócico & 0,419 & $0,317(0,020-5,135)$ & 0,419 & $0,317(0,020-5,135)$ & 0,715 & $0,637(0,057-7,130)$ \\
\hline & Ventosa & 0,998 & 0,000 & 0,998 & 0,000 & 0,998 & 0,000 \\
\hline & Fórceps & 0,088 & $8,471(0,725-98,943)$ & 0,335 & $4(0,239-67,075)$ & 0,999 & 0,000 \\
\hline & Espátulas & 0,999 & 0,000 & 0,999 & 0,000 & 0,999 & 0,000 \\
\hline \multicolumn{2}{|c|}{ Desgarro } & 0,925 & $1,099(0,153-7886)$ & 0,519 & $2,209(0,199-24,581)$ & 0,519 & $2,209(0,199-24,581)$ \\
\hline \multicolumn{2}{|c|}{ Episiotomía } & 0,460 & $2,103(0,293-15,119)$ & 0,973 & $1,043(0,094-11,616)$ & 0,996 & 0,000 \\
\hline \multicolumn{2}{|c|}{ Sobrepeso } & 0,937 & $1,102(0,099-12,307)$ & 0,575 & $2,215(0,137-35,798)$ & 0,995 & 35119018,51 \\
\hline \multicolumn{2}{|c|}{ Obesidad } & 0,654 & $1,737(0,155-19,495)$ & 0,379 & $3,492(0,215-56,668)$ & 0,995 & 27380929,69 \\
\hline \multicolumn{2}{|c|}{$\begin{array}{l}\text { Ganancia ponderal } \\
\text { excesiva }\end{array}$} & 0,278 & $3,522(0,363-34,188)$ & 0,995 & 30102015,700 & 0,995 & 30102015,54 \\
\hline
\end{tabular}

Entre paréntesis se muestran los intervalos de confianza al $95 \%$ para cada OR (Odds ratio) 
Tabla 4. Regresión logística binomial entre la disfunción sexual a los 6 meses, 12 meses y 7 años del parto y las variables independientes

\begin{tabular}{|c|c|c|c|c|c|c|c|}
\hline \multirow{2}{*}{\multicolumn{2}{|c|}{ Variable }} & \multicolumn{2}{|r|}{6 meses posparto } & \multicolumn{2}{|r|}{12 meses posparto } & \multicolumn{2}{|r|}{7 años posparto } \\
\hline & & $\mathrm{p}$ & OR & $\mathrm{p}$ & OR & $\mathrm{p}$ & OR \\
\hline \multicolumn{2}{|c|}{ Macrosomía } & 0,002 & $10,140(2,261-45,478)$ & 0,002 & $10,140(2,261-45,478)$ & 0,002 & $10,140(2,261-45,478)$ \\
\hline \multirow{4}{*}{$\begin{array}{l}\text { Tipo de } \\
\text { parto }\end{array}$} & Eutócico & 0,788 & $0,796(0,151-4,192)$ & 0,788 & $0,796(0,151-4,192)$ & 0,788 & $0,796(0,151-4,192)$ \\
\hline & Ventosa & 0,940 & $0,910(0,080-10,361)$ & 0,940 & $0,910(0,080-10,361)$ & 0,940 & $0,910(0,080-10,361)$ \\
\hline & Fórceps & 0,998 & 0,000 & 0,998 & 0,000 & 0,998 & 0,000 \\
\hline & Espátulas & 0,999 & 0,000 & 0,999 & 0,000 & 0,999 & 0,000 \\
\hline \multicolumn{2}{|c|}{ Desgarro } & 0,213 & $0,359(0,071-1,800)$ & 0,213 & $0,359(0,071-1,800)$ & 0,213 & $0,359(0,071-1,800)$ \\
\hline \multicolumn{2}{|c|}{ Episiotomía } & 0,293 & $2,123(0,522-8,640)$ & 0,757 & $1,257(0,295-5,352)$ & 0,757 & $1,257(0,295-5,352)$ \\
\hline \multicolumn{2}{|c|}{ Sobrepeso } & 0,671 & $1,478(0,243-8,997)$ & 0,427 & $2,228(0,309-16,064)$ & 0,427 & $2,228(0,309-16,064)$ \\
\hline \multicolumn{2}{|c|}{ Obesidad } & 0,125 & $3,579(0,703-18,212)$ & 0,024 & $7,321(1,307-41,006)$ & 0,024 & $7,321(1,307-41,006)$ \\
\hline \multicolumn{2}{|c|}{$\begin{array}{l}\text { Ganancia ponderal } \\
\text { excesiva }\end{array}$} & 0,122 & $3,570(0,711-17,934)$ & 0,047 & $8,427(1,026-69,225)$ & 0,047 & $8,427(1,026-69,225)$ \\
\hline \multicolumn{2}{|c|}{ Tiempo expulsivo } & 0,658 & $0,997(0,985-1,010)$ & 0,861 & $0,999(0,986-1,012)$ & 0,861 & $0,999(0,986-1,012)$ \\
\hline
\end{tabular}

Entre paréntesis se muestran los intervalos de confianza al $95 \%$ para cada OR (Odds ratio)

todos los periodos estudiados se observó una relación positiva entre la incontinencia de orina y el sobrepeso/ obesidad, en comparación con aquellas pacientes con IMC $<25$. Además, también se evidenció dicha relación entre este tipo de disfunción de suelo pélvico y una ganancia ponderal excesiva, si se compara con las pacientes que tuvieron una ganancia ponderal adecuada durante la gestación. Por su parte, el tiempo de expulsivo ha obtenido una OR de 1.

Posteriormente, se realizó el análisis de las mismas variables, pero tomando como variable dependiente la incontinencia fecal. Los resultados obtenidos mostraron una relación estadísticamente significativa entre la incontinencia fecal a los 7 años y la macrosomía fetal, con una OR 32,5 (IC 95 \% 2,8-373,9), no siendo así para la incontinencia fecal a los 6 meses y al año, aunque sí se observó una tendencia a que a mayor peso fetal mayor riesgo de incontinencia fecal. Para el resto de las variables, aún sin resultados estadísticamente significativos, cabe destacar la relación positiva entre el sobrepeso y la obesidad con la incontinencia fecal a los 6 y 12 meses del parto, así como con la ganancia de peso excesiva y la incontinencia fecal a los 6 meses. Atendiendo a la presencia de desgarro perineal, realización de episiotomía y tiempo de expulsivo no se obtuvieron resultados estadísticamente significativos en ninguno de los periodos de estudio, aunque sí se puede observar una tendencia a mayor de riesgo de incontinencia fecal a los 6 y 12 meses del parto eutócico e instrumental con fórceps.

Por último, se realizó el análisis de las mismas variables tomando como evento dependiente la disfunción sexual. Los resultados obtenidos no mostraron relación estadísticamente significativa entre esta variable y el tipo de parto, realización de episiotomía y tiempo de expulsivo, ni a los 6 meses, ni a los 12 meses ni a los 7 años. Sin embargo, sí hubo significación estadística entre la presencia de disfunción sexual y la macrosomía 


\section{DEFECTOS DEL SUELO PÉLVICO POSPARTO EN FUNCIÓN DEL ÍNDICE DE MASA CORPORAL PREGESTACIONAL Y GANANCIA PONDERAL DE LA MUJER DURANTE EL EMBARAZO}

fetal, obteniendo una OR 10,140 IC $95 \%(2,261$ $45,478)$ en los tres periodos estudiados. Así mismo, al año y a los 7 años del parto, la obesidad obtuvo una OR: 7,321 IC $95 \%(1,307-41,006)$ y la ganancia de peso excesiva una OR: 8,427 IC $95 \%(1,026-69,225)$.

\section{DISCUSIÓN}

Diversos estudios han demostrado que las disfunciones de suelo pélvico tienen una fisiopatología y un origen multifactorial. Así, trabajos como el realizado por Reimers y cols. (3) o Chan y cols. (8), evaluaron los factores de riesgo asociados al prolapso de órganos pélvicos, siendo el parto vaginal el que más se ha relacionado con estas disfunciones. Todos ellos defienden que aquellas mujeres en las que ya existía una alteración anatómica del suelo pélvico previa a la gestación fueron más propensas al desarrollo de disfunciones posparto del suelo pélvico y que el tipo de cambio acontecido durante la gestación (aumento del hiato genital, aumento de la movilidad de los órganos pélvicos, etc.) se relacionaba con los síntomas posparto de dichas disfunciones. Defienden que estos cambios pueden mejorar con la realización de ejercicios de suelo pélvico y rehabilitación. En este estudio, el factor de riesgo que más se relacionó tanto con la incontinencia urinaria como con la fecal fue la macrosomía fetal, no encontrándose asociación estadísticamente significativa en el resto variables estudiadas. Así mismo, los factores de riesgo más relacionados con la disfunción sexual fueron la macrosomía, la obesidad y la ganancia ponderal excesiva, encontrándose una asociación estadísticamente significativa entre estas variables a los 12 meses y a los 7 años del parto.

Atendiendo al tipo de parto como factor de riesgo para el desarrollo de incontinencia o prolapso de órganos pélvicos, se demostró que el parto vaginal, $\mathrm{y}$ en particular el parto instrumental, aumenta el riesgo de lesiones perineales que puedan desembocar en disfunciones a este nivel (9). En el presente estudio, sin obtener resultados estadísticamente significativos, sí se observó una asociación positiva entre la incontinencia de orina y el parto vaginal, en comparación con el parto por cesárea. Además, en la literatura, se puede encontrar otro estudio en el que se analizaron las tasas de incontinencia fecal tras parto vaginal en comparación con el parto por cesárea, obteniendo mayor tasa de incontinencia tras el parto vía vaginal $(0,37 \%$ vs. $0,22 \%)$. Sin embargo, destacan que el riesgo de incontinencia fecal no es nulo en las pacientes que culminan la gestación mediante cesárea, por lo que debe haber otros factores asociados (10). En este estudio no se observó relación entre la incontinencia fecal o la disfunción sexual y la vía de parto. Black y cols. (11), describieron las posibles complicaciones maternas asociadas al parto instrumental, especialmente al parto mediante fórceps, destacando las lesiones traumáticas perineales tales como el uso de episiotomía o los desgarros del esfínter anal. Comparado con la cesárea, el parto instrumental aumenta el riesgo de incontinencia urinaria y de prolapso de órganos pélvicos subsidiarios a reparación quirúrgica. En este estudio, no se evidenció asociación estadísticamente significativa entre la episiotomía o los desgarros perineales y las disfunciones del suelo pélvico. Sin embargo, los datos obtenidos hacen suponer que estas dos variables aumentan el riesgo de incontinencia de orina a los 6 y 12 meses posparto. Además, los resultados postulan que la presencia de desgarro perineal aumenta las tasas de incontinencia fecal y de disfunción sexual tanto a corto como a largo plazo, aunque no hubo asociación estadística respecto a los mismos. La macrosomía fetal también se ha definido como un factor de riesgo en las disfunciones de suelo pélvico. En el presente estudio, un peso fetal superior a $4000 \mathrm{~g}$ sí se asoció a una mayor tasa de incontinencia de orina a los 6 meses, 12 meses y 7 años posparto. Así mismo, se demostró una asociación estadísticamente significativa entre la presencia de macrosomía, la incontinencia fecal a los 7 años y la disfunción sexual (en los tres periodos estudiados).

Otro de los factores de riesgo que se han asociado de manera consistente a los defectos del suelo pélvico 
es la obesidad, ya que se ha demostrado que estas alteraciones son más prevalentes en mujeres con IMC mayor de 30 e incluso, que la gravedad de estos es mayor cuanto mayor es el IMC de la paciente $(7,12$, 13). Estos hallazgos también se mostraron en el estudio, obteniendo resultados estadísticamente significativos entre la obesidad y la incontinencia fecal a los 7 años y la disfunción sexual a los 12 meses y a los 7 años del parto. Sin llegar a la significación estadística, también se observó una asociación positiva entre la incontinencia urinaria y la presencia de sobrepeso y obesidad. Además, el IMC es una medida útil para estimar correctamente la ganancia óptima de peso durante la gestación. Esta ganancia ponderal tiende a ser menor a mayor IMC y depende del IMC previo al embarazo y de si se trata de una gestación única o gemelar. Asociaciones científicas como la SEGO recomiendan que en gestaciones únicas, la ganancia ponderal debe ser: entre 11,5 - $16 \mathrm{Kg}$ en pacientes con pesos normales (IMC 18,5 - 24,9); entre 7 y 11,5 Kg para mujeres con sobrepeso (25 - 29); y menor de 7 $\mathrm{Kg}$ para mujeres con obesidad (IMC $\geq 30$ ) (14). Hay estudios que han demostrado que la obesidad es un factor de riesgo independiente para las disfunciones de suelo pélvico y mantienen que el control del peso, así como una ganancia ponderal adecuada durante la gestación, parecen ser una parte importante en el asesoramiento y prevención de estos procesos (15). En este estudio se agruparon a las pacientes según hayan tenido una ganancia ponderal adecuada o no, entendiendo como no adecuada a una ganancia ponderal mayor a lo establecido por la SEGO. Se obtuvo que la ganancia ponderal excesiva se relacionó, de forma estadísticamente significativa, con mayores tasas de disfunción sexual al año y a los 7 años del parto, con una OR de 8,427 IC $95 \%(1,026-69,225)$ y que hubo una tendencia a la asociación positiva entre aquellas pacientes con ganancia ponderal superior a lo recomendado para su IMC previo y los tres tipos de disfunción de suelo pélvico, en los tres periodos estudiados. Se debe tener en cuenta que numerosos estudios han postulado que es más probable tener un feto macrosómico (en este estudio demostró una asociación clara con los defectos de suelo pélvico), si se parte de un IMC elevado o si la ganancia ponderal durante la gestación es excesiva, lo que potenciaría aún más el riesgo de padecer alguno de estos defectos (16).

Las disfunciones del suelo pélvico tienen un origen multifactorial y suelen ser secundarias a la conjunción de varios factores de riesgo que desencadenan el desarrollo de estos problemas. Como se indicó anteriormente, uno de los factores de riesgo más implicados en su patogenia es la gestación $(4,9$, 17). Bien conocido es que la rehabilitación del suelo pélvico es una medida protectora y a su vez, una forma de tratamiento. No obstante, muchas pacientes no la realizan por falta de conocimiento sobre el problema y sus consecuencias. Liu y cols. (17) demostraron que, pese a la alta prevalencia de las disfunciones del suelo pélvico en las pacientes jóvenes, el nivel de conocimiento de la población sobre estas dolencias es bajo, así como sobre su prevención y tratamiento mediante rehabilitación. Si las pacientes estuvieran más informadas podrían tomar conciencia de la importancia tanto de la realización de ejercicios para fortalecer el suelo pélvico como del control del peso durante la gestación y podría verse una reducción, por tanto, en la incidencia de estos problemas $(14,17)$.

Entre las ventajas del estudio destaca que no solo incluye datos del posparto inmediato, sino que se analizan los datos a los 7 años del parto, lo que permite estudiar los factores de riesgo asociados a los defectos de suelo pélvico a largo plazo, constituyendo uno de los pocos estudios que incluyen un intervalo tan amplio. Por otra parte, el seguimiento de las pacientes a tan largo plazo puede ser difícil, sobre todo en aquellas pacientes que no presentan problemas de salud. Otra limitación del estudio es que el número de pacientes que presentan los defectos estudiados es bajo. Sin embargo, pese a estas limitaciones, el presente estudio puede ejercer de base para otros estudios, con 


\section{DEFECTOS DEL SUELO PÉLVICO POSPARTO EN FUNCIÓN DEL ÍNDICE DE MASA CORPORAL PREGESTACIONAL Y GANANCIA PONDERAL DE LA MUJER DURANTE EL EMBARAZO}

mayor tamaño muestral, que permitan comprobar los resultados obtenidos, ya que estos concuerdan con los resultados descritos previamente en la literatura.

\section{CONCLUSIONES}

Se concluye que la gestación y el parto son factores de riesgo de las disfunciones de suelo pélvico, pues factores como la macrosomía fetal se han asociado al desarrollo de estos, tanto a corto plazo tras el parto como años después del mismo. Además, la presencia de obesidad previa al parto y la ganancia ponderal parece que sí pueden tener su efecto en la fisiopatología de estos defectos, pues se ha objetivado en este trabajo la relación entre la obesidad y la incontinencia de orina a los 7 años del parto, así como el riesgo aumentado de disfunción sexual a los 12 meses y a los 7 años posparto en mujeres con IMC $>30$ y en aquellas con una ganancia ponderal excesiva durante la gestación. Así, dado que las disfunciones de suelo pélvico son un problema con una alta prevalencia entre las mujeres y una gran afectación en la calidad de vida, sería conveniente la realización de nuevos estudios con el fin de poder obtener resultados significativos que demuestren la asociación de estos defectos con el IMC previo elevado y la ganancia ponderal excesiva durante la misma. No obstante, es de vital importancia informar a las pacientes, no solo de la necesidad de realizar rehabilitación de suelo pélvico para evitar estas dolencias, sino de que la pérdida de peso y el control de este durante la gestación son esenciales si se quieren prevenir defectos del suelo pélvico en un futuro.

\section{Sin conflictos de interés.}

\section{REFERENCIAS}

1. Zuchelo LTS, Bezerra IMP, Da Silva ATM, Gomes JM, Soares Júnior JM, Chada Baracat E, et al. Questionnaires to evaluate pelvic floor dysfunction in the postpartum period: a systematic review. Int $\mathrm{J}$ Womens Health. 2018; 10:409-424. doi: 10.2147/IJWH.S164266.
2. Colla C, Paiva LL, Ferla L, Trento MJB, de Vargas IMP, Dos Santos BA, et al. Pelvic floor dysfunction in the immediate puerperium, and 1 and 3 months after vaginal or cesarean delivery. Int J Gynaecol Obstet. 2018; 143(1):94-100. doi: 10.1002/ijgo.12561.

3. Reimers C, Siafarikas F, Stær-Jensen J, Småstuen MC, $\mathrm{B} ø \mathrm{~K}$, Ellström Engh M. Risk factors for anatomic pelvic organ prolapse at 6 weeks postpartum: a prospective observational study. Int Urogynecol J. 2019; 30(3):477482. doi: $10.1007 / \mathrm{s} 00192-018-3650-2$.

4. Urbankova I, Grohregin K, Hanacek J, Krcmar M, Feyereisl J, Deprest J, et al. The effect of the first vaginal birth on pelvic floor anatomy and dysfunction. Int Urogynecol J. 2019; 30(10):1689-1696. doi: 10.1007/s00192-019-04044-2.

5. Yang XJ, Sun Y. Comparison of caesarean section and vaginal delivery for pelvic floor function of parturients: a meta-analysis. Eur J Obstet Gynecol Reprod Biol. 2019; 235:42-48. doi: 10.1016/j.ejogrb.2019.02.003.

6. Pomian A, Lisik W, Kosieradzki M, Barcz E. Obesity and Pelvic Floor Disorders: A Review of the Literature. Med Sci Monit. 2016; 22:1880-1886. doi: 10.12659/ msm. 896331.

7. Rechberger T, Nowakowski $Ł$, Rechberger E, Ziętek A, Winkler I, Miotła P. Prevalence of common comorbidities among urogynaecological patients. Ginekol Pol. 2016; 87(5):342-346. doi: 10.5603/ GP.2016.0012.

8. Chan SS, Choy KW, Yiu KW, Cheung RY, Leung TY. Pelvic floor disorders related to pregnancy: a prospective observational study. Hong Kong Med J [Internet]. 2017 [consultado marzo de 2020]; 23(Supp12):42-46. Disponible en: https://www.hkmj.org/system/files/ hkm1706sp2p42.pdf

9. Blomquist JL, Muñoz A, Carroll M, Handa VL. Association of delivery mode with pelvic floor disorders after childbirth. JAMA. 2018; 320(23):2438-2447. doi: 10.1001/jama.2018.18315.

10. Larsson C, Hedberg CL, Lundgren E, Söderström L, TunÓn K, Nordin P. Anal incontinence after caesarean and vaginal delivery in Sweden: a national populationbased study. Lancet. 2019; 393(10177):1233-1239. doi: 10.1016/S0140-6736(18)32002-6.

11. Black M, Murphy DJ. Forceps delivery for nonrotational and rotational operative vaginal delivery. Best Pract Res Clin Obstet Gynaecol. 2019; 56:55-68. doi: 10.1016/j.bpobgyn.2019.02.002. 
12. Nygaard CC, Schreiner L, Morsch TP, Saadi RP, Figueiredo MF, Padoin AV. Urinary Incontinence and Quality of Life in Female Patients with Obesity. Rev Bras Ginecol Obstet. 2018; 40(9):534-539. doi: 10.1055/s-0038-1670626.

13. Mohd Yusoff D, Awang S, Kueh YC. Urinary incontinence among pregnant women attending an antenatal clinic at a tertiary teaching hospital in NorthEast Malaysia. J Taibah Univ Med Sci. 2018; 14(1):3946. doi: 10.1016/j.jtumed.2018.11.009.

14. Sociedad Española de Ginecología y Obstetricia. Guía de asistencia práctica. Control prenatal del embarazo normal. Prog Obstet Ginecol. 2018; 61(05):510-527. DOI: 10.20960/j.pog.00141

15. Bodner-Adler B, Kimberger O, Laml T, Halpern K, Beitl C, Umek W, et al. Prevalence and risk factors for pelvic floor disorders during early and late pregnancy in a cohort of Austrian women. Arch Gynecol Obstet. 2019; 300(5):1325-1330. doi: 10.1007/s00404-01905311-9.

16. Agudelo-Espitia V, Parra-Sosa BE, Restrepo-Mesa SL. Factors associated with fetal macrosomia. Rev Saude Publica. 2019; 53:100. doi: 10.11606/s15188787.2019053001269.

17. Liu J, Tan SQ, Han HC. Knowledge of pelvic floor disorder in pregnancy. Int Urogynecol J. 2019; 30(6):991-1001. doi: 10.1007/s00192-019-03891-3.

Recibido 8 de marzo de 2021

Aprobado 10 de junio de 2021 